\title{
ERRATA
}

\section{COMMENT ON THE CONSTRAINT FOR ANOMALOUS JACOBI IDENTITY}

[MOD. PHY. LETT. A, Vol. 1, No. 2 (1986) 103-110]

BO-YU HUO and YAO-ZHONG ZHANG

Equation (18) on page 107 contains some errors. It should read

$$
\begin{aligned}
& {\left[G^{a}(x), Q^{b c d}(q ; y, z, u)\right]-\left[G^{b}(y), Q^{c d a}(q ; z, u, x)\right]+\left[G^{c}(z), Q^{d a b}(q ; u, x, y)\right]} \\
& \quad-\left[G^{d}(u), Q^{a b c}(q ; x, y, z)\right]-f^{a b e} Q^{e c d}(q ; x, z, u) \delta(x-y)+f^{b c e} Q^{e d a}(q ; y, u, x) \\
& \quad \times \delta(y-z)-f^{c d e} Q^{e a b}(q ; u, x, y) \delta(z-u)+f^{d a e} Q^{e b c}(q ; u, y, z) \delta(u-x) \\
& \quad-f^{a c e} Q^{e d b}(q ; x, u, y) \delta(x-z)+f^{b d e} Q^{e a c}(q ; y, x, z) \delta(y-u)=0 .
\end{aligned}
$$

Correspondingly, Eq. (19) should read

$$
\begin{aligned}
& {\left[G^{a}(x),\left[G^{b}(y),\left[G^{c}(z), G^{d}(u)\right]\right]\right]+(\text { perm. }) } \\
&= f^{a b e} \delta(x-y)\left\{\left[G^{e}(x),\left[G^{c}(z), G^{d}(u)\right]\right]+(\text { perm. })\right\} \\
&-f^{b c e} \delta(y-z)\left\{\left[G^{e}(y),\left[G^{d}(u), G^{a}(x)\right]\right]+(\text { perm. })\right\} \\
&+f^{c d e} \delta(z-u)\left\{\left[G^{e}(u),\left[G^{a}(x), G^{b}(y)\right]\right]+(\text { perm. })\right\} \\
&-f^{d a e} \delta(u-x)\left\{\left[G^{e}(u),\left[G^{b}(y), G^{c}(z)\right]\right]+(\text { perm. })\right\} \\
&+f^{a c e} \delta(x-z)\left\{\left[G^{a}(x),\left[G^{d}(u), G^{b}(y)\right]\right]+(\text { perm. })\right\} \\
&-f^{b d e} \delta(y-u)\left\{\left[G^{e}(y),\left[G^{a}(x), G^{c}(z)\right]\right]+(\text { perm. })\right\}
\end{aligned}
$$

